\title{
A CORE-ENVELOPE DECOUPLING DYNAMO MODEL FOR CATACLYSMIC VARIABLES
}

\author{
L. ZANGRILLI ${ }^{1}$, C. A. TOUT ${ }^{2}$, A. BIANCHINI ${ }^{1}$ \\ 1. Department of Astronomy, University of Padova \\ Vicolo dell'Ossevatorio 5, 35122 Padova, Italy \\ 2. Institute of Astronomy, University of Cambridge \\ Madingley Road, Cambridge CBз OHA, UK
}

The progenitors of cataclysmic variables (CVs) undergo a common envelope phase, during which their initial orbital period is reduced to a few hours. After this phase the radiative core of the secondary might rotate at a lower rate than the tidally coupled envelope. A shear is then generated at the core-envelope interface which can give rise to a boundary layer dynamo and, consequently, to the magnetic braking of the binary system. The temporal variation of the shear energy content can be obtained by comparing the rate of magnetic energy production with the input rate of orbital energy in the convective envelope. We assume for the flux tubes a filling factor, $f_{\mathrm{V}}$, owing to the expected size of the tubes and the fact that the dynamo action may be concentrated inside an equatorial belt of the boundary layer shell. We also assume that the production of toroidal field is efficient only inside the boundary layer, while the poloidal component of the field can be enhanced also across the outer convective envelope (see also Zangrilli \& Bianchini 1995). We find that the decrease in the shear energy, caused by the continuous production of magnetic field and by the progressive reduction of the core moment of inertia, leads the core coupling with the envelope, thus switching off the dynamo at the core-envelope boundary. Further details of this core-envelope decoupling dynamo model will be given in a forthcoming paper (Zangrilli, Tout \& Bianchini 1996).

\begin{tabular}{cccc}
\multicolumn{4}{c}{ Table 1 } \\
\hline Model & Period $_{\text {core }}($ initial $)$ & Period $_{\text {env }}($ initial $)$ & $f_{\mathrm{V}}$ \\
\hline MOD1 & $100 \mathrm{~h}$ & $6 \mathrm{~h}$ & 0.1 \\
MOD2 & $100 \mathrm{~h}$ & $6 \mathrm{~h}$ & 0.01 \\
MOD3 & $10 \mathrm{~h}$ & $6 \mathrm{~h}$ & 0.01 \\
\hline
\end{tabular}

The evolution of a CV has been computed using a double polytrope code for the secondary star (Rappaport, Verbunt \& Joss 1983) and the magnetic 

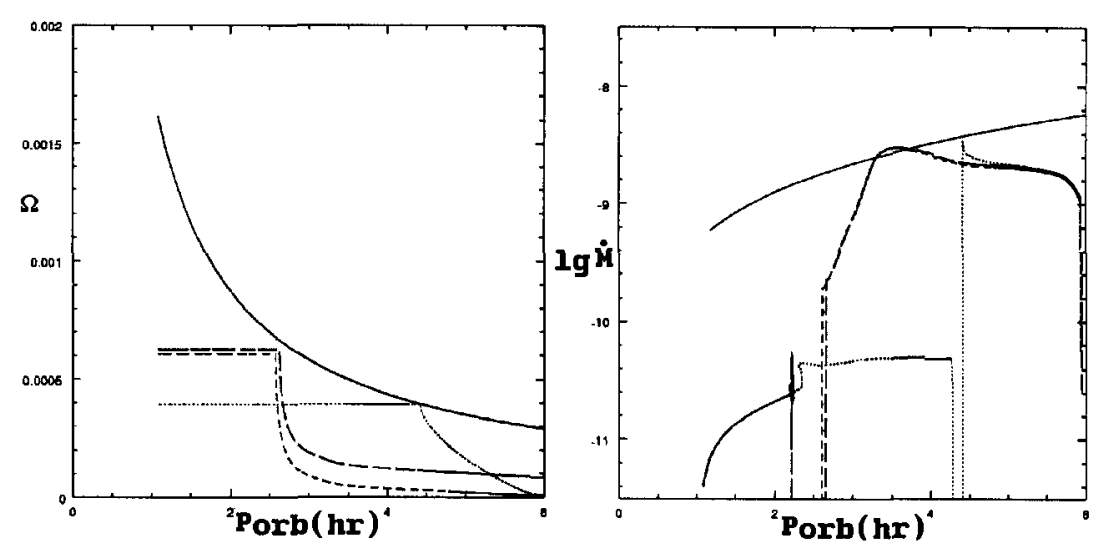

Figure 1. (a: left) Core angular velocity evolution versus orbital period. Dotted line corresponds to MOD1; long dashed and short dashed lines correspond to MOD2 and MOD3, respectively. Continuous line is the envelope angular velocity, which is the same for the three models. (b: right) Mass transfer rate versus orbital period. Line styles are as in (a). Continuous line is the critical mass transfer rate curve.

braking model of Mestel \& Spruit (1987). The evolutionary tracks shown in Fig. 1 refer to the three models of Table 1. In Fig. 1a we see that, in MOD1, the core couples with the envelope at about $4.5 \mathrm{~h}$, thus switching off the dynamo. This is caused by the large magnetic energy production, favoured by a large filling factor. In MOD2 and MOD3 this happens at just about $3 \mathrm{~h}$, owing to the small moment of inertia of the core at this stage. In Fig. $1 \mathrm{~b}$ we see that in MOD1 mass transfer ceases much earlier than the $3 \mathrm{~h}$ upper limit of the gap. Instead, MOD2 and MOD3 reproduce it well. Moreover, we can see that both MOD2 and MOD3, in the interval $3 \ldots 3.5 \mathrm{~h}$, are above the critical mass transfer curve (Shafter 1992), which separates stable accretion discs (above) from the unstable ones (below). This appears to be in agreement with the observed lack of dwarf nova systems in this interval of orbital periods. It is worth noticing that $f_{\mathrm{V}}=0.01$ gives the better agreement with the observed statistics of CVs, while little dependence of the binary evolution on the initial rotational regime of the core is found.

\section{References}

Mestel, L., Spruit, H.C., 1987, MNRAS, 226, 57

Rappaport, S., Verbunt, F., Joss, P.C., 1983, Ap. J., 275, 713

Shafter, A.W., 1992, Ap. J., 394, 268

Zangrilli, L., Bianchini, A., 1995, in "Cataclysmic Variables", eds A. Bianchini et al., Kluwer, p405

Zangrilli, L., Tout, C.A., Bianchini, A., 1996, in preparation 